\title{
Biofilm formation by virulent and non-virulent strains of Haemophilus parasuis
}

\author{
Bernardo Bello-Ortí ${ }^{1 \dagger}$, Vincent Deslandes ${ }^{2 \dagger}$, Yannick DN Tremblay ${ }^{2}$, Josée Labrie ${ }^{2}$, Kate J Howell ${ }^{3}$, \\ Alexander W Tucker ${ }^{3}$, Duncan J Maskell ${ }^{3}$, Virginia Aragon ${ }^{1,4}$ and Mario Jacques ${ }^{2^{*}}$
}

\begin{abstract}
Haemophilus parasuis is a commensal bacterium of the upper respiratory tract of healthy pigs. It is also the etiological agent of Glässer's disease, a systemic disease characterized by polyarthritis, fibrinous polyserositis and meningitis, which causes high morbidity and mortality in piglets. The aim of this study was to evaluate biofilm formation by well-characterized virulent and non-virulent strains of $H$. parasuis. We observed that non-virulent strains isolated from the nasal cavities of healthy pigs formed significantly $(p<0.05)$ more biofilms than virulent strains isolated from lesions of pigs with Glässer's disease. These differences were observed when biofilms were formed in microtiter plates under static conditions or formed in the presence of shear force in a drip-flow apparatus or a microfluidic system. Confocal laser scanning microscopy using different fluorescent probes on a representative subset of strains indicated that the biofilm matrix contains poly-N-acetylglucosamine, proteins and eDNA. The biofilm matrix was highly sensitive to degradation by proteinase K. Comparison of transcriptional profiles of biofilm and planktonic cells of the non-virulent H. parasuis F9 strain revealed a significant number of up-regulated membrane-related genes in biofilms, and genes previously identified in Actinobacillus pleuropneumoniae biofilms. Our data indicate that non-virulent strains of $H$. parasuis have the ability to form robust biofilms in contrast to virulent, systemic strains. Biofilm formation might therefore allow the non-virulent strains to colonize and persist in the upper respiratory tract of pigs. Conversely, the planktonic state of the virulent strains might allow them to disseminate within the host.
\end{abstract}

\section{Introduction}

Haemophilus parasuis is a Gram-negative bacterium and a commensal organism of the upper respiratory tract of healthy pigs. It is also the etiological agent of Glässer's disease, a systemic disease characterized by polyarthritis, fibrinous polyserositis and meningitis, which causes high morbidity and mortality in piglets [1]. Glasser's disease is recognized as one of the main causes of economic loss in the pig industry.

The heterogeneity among $H$. parasuis strains has been demonstrated by multiple methods, including multilocus sequence typing (MLST) which has shown a genetic lineage associated with polyserositis (cluster A) and another lineage associated with nasal colonization (cluster C)

\footnotetext{
* Correspondence: mario.jacques@umontreal.ca

${ }^{\dagger}$ Equal contributors

${ }^{2}$ Groupe de recherche sur les maladies infectieuses du porc, Faculté de médecine vétérinaire, Université de Montréal, St-Hyacinthe, Québec J2S 7C6, Canada

Full list of author information is available at the end of the article
}

[2]. Serum resistance [3], phagocytosis resistance [4], and invasion of endothelial cells $[5,6]$ have been associated with virulence of $H$. parasuis. Some putative virulence factors have been reported $[7,8]$. Those include the capsule [4], lipooligosaccharide (LOS) [9-11] and other genes involved in polysaccharide production, such as galE and gall, which have been associated with serum resistance and biofilm production [12]. Genes involved in sialic acid utilization were identified as potential virulence factors [13]. The sialytransferase gene $l s g B$ was predominantly present in systemic isolates and not in nasal isolates, and sialylation of the LOS was observed in the virulent strain $H$. parasuis Nagasaki. In addition, a family of trimeric autotransporters, designated virulence associated trimeric autotransporters (VtaA) has been described $[14,15]$ and these can be divided into three groups based on their translocation domains [14]. Group $3 v t a A$ gene is highly conserved among invasive and non-invasive strains, whilst 
groups 1 and $2 v t a A$ genes were detected only in virulent strains.

Bacterial biofilms are structured clusters of bacterial cells enclosed in a self-produced polymer matrix that are attached to a surface [16-18]. Bacteria can adhere to biotic surfaces (e.g. cells at the mucous layer) as well as abiotic surfaces (e.g. floor or equipment found at a farm). The polymer matrix is often composed of exopolysaccharides, proteins and nucleic acids. The biofilm protects bacteria from hostile environmental conditions. Bacteria within a biofilm can resist attack from the host immune response, and are less sensitive than planktonic cells to desiccation and to the action of biocides. Biofilm formation by nasal strains of $H$. parasuis has been previously reported [19]. Although the role of biofilm in $H$. parasuis pathogenesis is not clear, expression of genes with putative function in biofilm formation was detected during pulmonary infection [20]. The aims of this study were to compare biofilm formation by well-characterized virulent and non-virulent strains of $H$. parasuis and to analyse the gene expression during biofilm growth. Such analyses would help explore the possible role of biofilms in the pathogenesis of $H$. parasuis.

\section{Materials and methods}

\section{Bacterial strains}

The $H$. parasuis strains used in this study and their main phenotypic and genotypic characteristics are listed in Table 1. Bacteria were grown on Brain Heart Infusion (BHI; Oxoid Ltd, Basingstoke, Hampshire, UK) agar containing $10 \mu \mathrm{g} / \mathrm{mL}$ NAD or on chocolate agar (Biomerieux, Madrid, Spain). Plates were incubated overnight at $37{ }^{\circ} \mathrm{C}$ with $5 \% \mathrm{CO}_{2}$.

\section{Biofilm assays \\ Static conditions}

Biofilms were cultured in 96-well microtiter plates as described by Wu et al. [21], with some modifications. Briefly, colonies from overnight agar cultures were resuspended in BHI-NAD containing $5 \mu \mathrm{g} / \mathrm{mL} \mathrm{NAD}$, and the suspension was aliquoted $(100 \mu \mathrm{L})$ in triplicate in a flat-bottom 96well polystyrene plate (Costar ${ }^{\bullet} 3599$, Corning, NY, USA) and incubated for $40 \mathrm{~h}$ at $37{ }^{\circ} \mathrm{C}$. Wells containing sterile broth were used as negative control. Following incubation, biofilms were treated as described before [22] with some modifications. Briefly, the liquid medium was removed using a vacuum and unattached cells were removed by immersing the plate once in MilliQ water. The water was removed with a vacuum and excess water was removed by inverting plates onto a paper towel. Biofilms were then stained with $0.1 \%(\mathrm{w} / \mathrm{v})$ crystal violet for $2 \mathrm{~min}$. Biofilms were washed once with distilled water and then dried at $37{ }^{\circ} \mathrm{C}$ for $15 \mathrm{~min}$. The stain was then released with $100 \mu \mathrm{L}$ of $70 \%(\mathrm{v} / \mathrm{v})$ ethanol and the amount of released stain was
Table 1 Haemophilus parasuis strains used in the present study

\begin{tabular}{|c|c|c|c|c|c|c|}
\hline \multirow[t]{2}{*}{ Strains } & \multicolumn{2}{|c|}{ Susceptibility ${ }^{1}$ to } & \multirow[t]{2}{*}{$\operatorname{lsg} B^{2}$} & \multirow{2}{*}{$\begin{array}{l}\text { group1 } \\
\text { vtaA } A^{2}\end{array}$} & \multirow[t]{2}{*}{$\mathrm{MLST}^{3}$} & \multirow[t]{2}{*}{ Serova } \\
\hline & Serum & Phagocytosis & & & & \\
\hline
\end{tabular}

Nasal strains

\begin{tabular}{|c|c|c|c|c|c|c|}
\hline F9 & S & $\mathrm{S}$ & - & - & C & 6 \\
\hline ND14-1 & S & $\mathrm{S}$ & - & - & C & 7 \\
\hline SC14-1 & $S$ & $S$ & - & - & C & 15 \\
\hline MU21-2 & S & $S$ & - & - & C & 7 \\
\hline$S L 3-2$ & S & $S$ & - & + & B & 10 \\
\hline \multicolumn{7}{|c|}{ Strains from lesions } \\
\hline Nagasaki & $\mathrm{R}$ & $\mathrm{R}$ & + & + & A & 5 \\
\hline P015/96 & $\mathrm{R}$ & $\mathrm{R}$ & + & + & A & 5 \\
\hline ER-6P & $\mathrm{R}$ & $\mathrm{R}$ & + & + & A & 15 \\
\hline 264/99 & $R$ & $\mathrm{R}$ & + & + & A & 10 \\
\hline IT29205 & $\mathrm{R}$ & $\mathrm{R}$ & + & + & A & 4 \\
\hline 2725 & S & $\mathrm{R}$ & + & + & A & 10 \\
\hline 373/03A & 1 & $\mathrm{R}$ & - & + & A & 7 \\
\hline PV1-12 & $\mathrm{S}$ & $\mathrm{R}$ & - & + & B & 15 \\
\hline 9904108 & $S$ & $\mathrm{R}$ & - & - & C & 4 \\
\hline
\end{tabular}

${ }^{1}(\mathrm{~S})$ sensitive; (I) intermediate; $(\mathrm{R})$ resistant.

${ }^{2}(+)$ gene present; $(-)$ gene absent.

${ }^{3}$ Cluster associated with isolates from systemic lesions (A); with nasal isolation

(C); or cluster with no statistical association with clinical origin (B) [2].

quantified by measuring the absorbance at $590 \mathrm{~nm}$ with a microplate reader (Powerwave; BioTek Instruments, Winooski, VT, USA).

\section{Shear force conditions}

Biofilms were cultured in a drip-flow apparatus (DFR 110 Biofilm Reactor, BioSurface Technologies Corp. Bozeman, MT, USA) as described by Goeres et al. [23] with some modifications [24]. Briefly, colonies of $H$. parasuis strains F9, MU21-2, ER-6P or Nagasaki on BHI-NAD agar were resuspended into $13 \mathrm{~mL}$ of fresh $\mathrm{BHI}-\mathrm{NAD}$ to an $\mathrm{OD}_{600}$ of 0.1 and $12.5 \mathrm{~mL}$ of this inoculum was transferred into a channel containing a glass slide (Catalogue \#48300-025, VWR, Ville Mont-Royal, QC, Canada). The apparatus was incubated for $24 \mathrm{~h}$ at $37{ }^{\circ} \mathrm{C}$ with $5 \% \mathrm{CO}_{2}$ to allow the biofilm to form under static conditions. The apparatus legs were then attached to create a $10^{\circ}$ downward slope. The apparatus was then connected to the nutrient system containing pre-warmed $\left(37^{\circ} \mathrm{C}\right) \mathrm{BHI}-\mathrm{NAD}$. The flow $(\sim 25 \mathrm{~mL}$ per hour per channel) of the medium was then initiated and maintained for $24 \mathrm{~h}$ at $37^{\circ} \mathrm{C}$. After $24 \mathrm{~h}$, the glass slide was removed and gently washed once with sterile MiliQ water. The biofilms were resuspended in $1.5 \mathrm{~mL}$ of MiliQ water, centrifuged and dried with a DNA 120 Speed $\mathrm{Vac}^{\circ}$ (Thermo Scientific, Ottawa, ON, Canada). The weight of dry biofilms was then measured. 
Biofilms were also cultured in a microfluidic system. Growth of biofilms in the BioFlux 200 device (Fluxion Biosciences, South San Francisco, CA, USA) was adapted from Benoit et al. [25] and the manufacturer's recommendations. Briefly, colonies of $H$. parasuis strains F9, MU212, ER-6P or Nagasaki on BHI-NAD agar were resuspended into $2.5 \mathrm{~mL}$ of fresh BHI-NAD to an $\mathrm{OD}_{600}$ of 1.0 and this suspension was serially diluted to $\mathrm{OD}_{600}$ of 0.5 and 0.25 . The microfluidic channels were wetted with BHI-NAD and were then inoculated with different bacterial suspensions $\left(\mathrm{OD}_{600} 1.0,0.5\right.$ or 0.25$)$. The microfluidic plate was incubated for $2 \mathrm{~h}$ at $37^{\circ} \mathrm{C}$ to allow bacteria to bind to the surface. The flow of fresh medium was then initiated and was set from $0.5 \mathrm{dyne} / \mathrm{cm}^{2}$ to $1.0 \mathrm{dyne} / \mathrm{cm}^{2}$. Growth of the biofilms was monitored for up to $24 \mathrm{~h}$ and, in some cases, fresh medium was added and the "waste" outlet was emptied to ensure that wells would not dry or spill. Images of BioFlux biofilms were obtained using an inverted fluorescence microscope (Olympus CKX41, Markham, ON, Canada), a digital camera (Retiga EX; Q Imaging, Surrey, $\mathrm{BC}$, Canada), and the software provided with the BioFlux 200 device.

\section{Confocal laser scanning microscopy (CLSM)}

Biofilms were prepared under static conditions as described above and were stained with FilmTracer ${ }^{\mathrm{Ts}}$ FM $^{\ominus} 1-43$ Green biofilm cell stain (Molecular Probes, Eugene, OR, USA) according to manufacturer's instructions. To determine the composition of the biofilm matrix, biofilms were stained with Wheat Germ Agglutinin (WGA-Oregon Green 488, Molecular Probes; binds to N-acetyl-Dglucosamine and $\mathrm{N}$-acetylneuraminic acid residues), FilmTracer $^{\mathrm{Tm}} \mathrm{SYPRO}^{\circ}$ Ruby biofilm matrix stain (Molecular Probes; labels most classes of proteins) or $\mathrm{BOBO}^{\mathrm{tm}}-3$ iodide (Molecular Probes; stains extracellular DNA) according to manufacturer's instructions. After a $30 \mathrm{~min}$ incubation at room temperature, the fluorescent marker solution was removed, biofilms were washed with water and the wells were then filled with $100 \mu \mathrm{L}$ of water or PBS for WGAstained biofilms. Stained biofilms were visualized by CLSM (Olympus FV1000 IX81, Markham, ON, Canada).

\section{Dispersion of biofilm by enzymatic treatments}

A biofilm dispersion assay was performed as described previously [26]. Briefly, biofilms were grown under static conditions as described above, and after the $40 \mathrm{~h}$ incubation, $50 \mu \mathrm{L}$ of DNase I $(500 \mu \mathrm{g} / \mathrm{mL}$ in $150 \mathrm{mM} \mathrm{NaCl}$, $\left.1 \mathrm{mM} \mathrm{CaCl}_{2}\right), 50 \mu \mathrm{L}$ of dispersin B $(100 \mu \mathrm{g} / \mathrm{mL}$ in PBS; Kane Biotech Inc., Winnipeg, $\mathrm{MB}$, Canada), or $50 \mu \mathrm{L}$ of

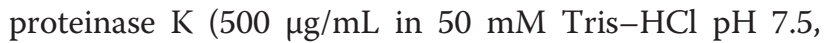
$1 \mathrm{mM} \mathrm{CaCl}_{2}$ ) were added directly to the biofilms. Control wells were treated with $50 \mu \mathrm{L}$ of the buffer without the enzyme. Wells treated with dispersin B were incubated for $5 \mathrm{~min}$ at $37^{\circ} \mathrm{C}$, and those treated with proteinase $\mathrm{K}$ or
DNase I were incubated for $1 \mathrm{~h}$ at $37{ }^{\circ} \mathrm{C}$. After the treatments, the biofilms were stained with crystal violet as described above.

\section{Effect of fibrinogen and fibronectin on biofilm formation} Biofilms were prepared under static conditions as described above with some modifications. Prior to inoculation, various concentrations of fibrinogen (bovine, porcine or human) $(0.5,1,2$ and $5 \mathrm{mg} / \mathrm{mL}$ ) or fibronectin (human) $(0.5,1$ and $2 \mathrm{mg} / \mathrm{mL})$ were added to the biofilm medium. Plates were prepared in duplicate for each experiment: one plate was used to measure biofilm formation and the other plate was used to measure growth of the bacteria in the presence of proteins. Both plates were incubated and one plate was stained as described before. The unstained replicate plate was used to evaluate growth by measuring the absorbance at $600 \mathrm{~nm}$.

\section{Genome sequencing and assembly}

Genomic DNA of the high biofilm producer strain F9 was prepared using the Blood and Tissue DNeasy kit (Qiagen) according to the manufacturer's instructions. Illumina libraries were prepared using $500 \mathrm{ng}$ of genomic DNA and modified Illumina protocols [27,28]. Paired-end sequencing was performed on an Illumina HiSeq 2000 analyzer for 75 cycles at the Wellcome Trust Sanger Institute (Hinxton, Cambridge, UK). The fastq reads were mapped to the complete SH0165 genome using Stampy to check for quality and purity of the sequence before any analyses [29,30].

A custom-made bioinformatics pipeline was used to assemble the draft genome sequence. Cutadapt was used to remove the adaptor sequences from the sequence reads that were previously introduced during the library preparation [31]. Any undetermined nucleotides (N's) were removed from reads and the program sickle [32] was used to trim low-quality sequences found at the ends of paired-end sequence reads resulting in a minimum length of $31 \mathrm{bp}$, using the program's default quality thresholds for the reads. Next, a custom Perl script was used to screen out low quality fastq reads and produce a single fastq file containing the good paired reads, and a separate file containing good quality single reads. Finally, we used Velvet [33] and VelvetOptimiser 2.2.0 [34] to assemble the fastq files into the de-novo genome assembly, made up of contiguous sequences (contigs). Assembly parameters were optimised to produce the highest quality assembly (i.e. highest n50 value) using VelvetOptimiser, which runs through all possible k-mer values from 19 to 71 in increments of 2 . The draft genome sequence was annotated using the automatic annotation software Prokka [35], including the rfam option. This Whole Genome Shogun project has been deposited [DDBJ/EMBL/GenBank: JHQI00000000]. The version described in this paper is version JHQI01000000. 


\section{Transcriptomics analysis}

The high biofilm producer strain F9 was chosen for transcriptomic analysis. Planktonic and biofilm samples were obtained under static conditions as described above with some modifications. Bacteria were cultured in 6well microtiter plates and after $36 \mathrm{~h}$, planktonic cultures were transferred to sterile tubes, whereas biofilm bacteria were collected by scraping the surface of the wells with lysis buffer (2\% SDS in PBS). Bacteria from both samples, biofilm or planktonic, were recovered by centrifugation and pellets were used for RNA extraction. For comparison, F9 was grown with shaking (220 rpm) until the culture reached stationary phase and this culture was then processed for RNA extraction. This is considered to be the "stationary culture" sample.

To extract RNA, bacterial pellets were resuspended in 2\% SDS, $16 \mathrm{mM}$ EDTA, $10 \mathrm{mM}$ Tris ( $\mathrm{pH}$ 8.0) and incubated for $5 \mathrm{~min}$ at $100{ }^{\circ} \mathrm{C}$. Afterwards, samples were processed by two hot acid phenol-chloroform extractions, followed by two chloroform/isoamyl alcohol extractions. RNA was then precipitated with 0.6 volumes of isopropanol, 0.1 volumes of $5 \mathrm{M}$ ammonium acetate and $1 \mu \mathrm{L}$ of glycogen. After centrifugation, the pellet was washed with $70 \%$ ethanol, dried and resuspended in warmed RNase-free water. To ensure that contaminating bacterial DNA was eliminated from the samples, treatment with RNase-free DNase (Qiagen) was performed. In addition, ribosomal RNA was eliminated with the Ribo-Zero rRNA removal kit (Epicentre Biotechnologies, Madison, WI, USA) following manufacturer instructions. PCR reactions using primers specific for $H$. parasuis 16S rRNA gene [36] were carried out to ensure that no bacterial DNA was left in the sample. Final RNA quality was verified with a Nanodrop spectrophotometer and the integrity was analyzed using Agilent Bioanalyzer 2100 (Agilent technologies). Libraries were generated using an Ion Torrent RNA-Seq v2 kit (Life Technologies) and sequenced using an Ion Torrent PGM (Life Technologies) with an Ion 318 chip (Life technologies) at the Centre for Research in Agricultural Genomics (CRAG, Campus de Bellaterra-UAB, Spain).

Bioinformatic analysis was performed following the count-based differential expression method. Briefly, reads quality control was performed using FastQC [37] and FASTX-Toolkit [38], and reads were then mapped to $H$. parasuis F9 genome using the recommended Torrent Mapping Alignment Program (TMAP) v3.4.1 with map2 setting [39]. Alignments were inspected using SAMtools [40] and Integrative Genomics Viewer (IGV) [41]. HTSeq v0.5.4p3 [42] was used for feature counting with intersection-nonempty setting and discarding nonprotein coding CDS. Differentially expressed genes (DEGs) were identified with edgeR $\mathrm{R}$ package [43] with a $5 \% P$-value cut-off, using an assigned dispersion value of 0.04 .
To perform gene set enrichments, a custom Gene Ontology (GO) database was built. Protein coding genes were BLASTed using BLASTp (version 2.2.28) against the non-redundant NCBI database (April 2014), e value of $10^{-3}$ and keeping first 20 hits. GO terms were mapped to Blast hits using Blast2GO [44]. The most significantly upregulated genes $(P<0.05)$ were identified as candidates for classic Fisher's exact testing through Blast2GO. Tests were performed for biological process (BP), cellular component (CC) and molecular function (MF) with $P<0.05$. Conservation of membrane-related genes among all $14 \mathrm{H}$. parasuis sequences available in GenBank [GenBank: APCA0 0000000.1, APBW00000000.1, ABKM00000000.2, APBX0 0000000.1, AZQU00000000.1, APBZ00000000.1, AOSU00 000000.1 , APBY00000000.1, APBV00000000.1, CP001321. 1, APBT00000000.1, APBU00000000.1, APCB00000000.1, CP005384.1] was achieved as follows: whole F9 proteome was analyzed using Phobius [45] via Blast2GO, positively predicted membrane-related genes were blasted against the $14 H$. parasuis genomic sequences using tBLASTn with the following settings: e value of $10^{-5}$, alignment length $>70 \%$ and match identity $>60 \%$. Whole-genome BLASTp comparisons were performed between $H$. parasuis and Actinobacillus pleuroneumoniae serovar 5b strain L20 [GenBank: NC_009053.1], using the following settings: e value of $10^{-5}$, alignment length $>90 \%$ and match identity $>40 \%$. All transcriptomic data were deposited in the Gene Expression Omnibus database [GEO: GSE56428].

\section{Results}

\section{Biofilm formation under static conditions}

The phenotypic and genotypic characteristics of the $H$. parasuis strains used in the present study are given in Table 1. Five non-virulent strains were recovered from the nasal cavities of healthy pigs while nine virulent strains were recovered from lesions of pigs with Glässer's disease. The ability of $H$. parasuis to form biofilms at the solidliquid interface in polystyrene microtiter plates was determined for each of the virulent and non-virulent strains (Figure 1A). Interestingly, the nasal, non-virulent strains formed significantly $(p<0.05)$ more biofilms than the virulent strains isolated from lesions of pigs with Glässer's disease (Figure 1B). We also noticed that biofilm production was stronger in strains sensitive to serum compared to resistant strains (Additional file 1A, $p=0.059$ ); in strains negative for $v t a A$ group 1 genes compared to strains positive for these genes (Additional file $1 \mathrm{~B}, p=0.189$ ); in strains belonging to MLST cluster C compared to strains belonging to MLST cluster A (Additional file $1 C, p=0.202$ ), and in strains negative for the sialyltransferase gene $l \mathrm{sg} B \mathrm{com}$ pared to strains positive for $l \operatorname{sg} B$ (Additional file $1 \mathrm{D}, p=$ 0.228). These differences however were not statistically significant probably due to the small number of strains in some of the groups that were compared. 

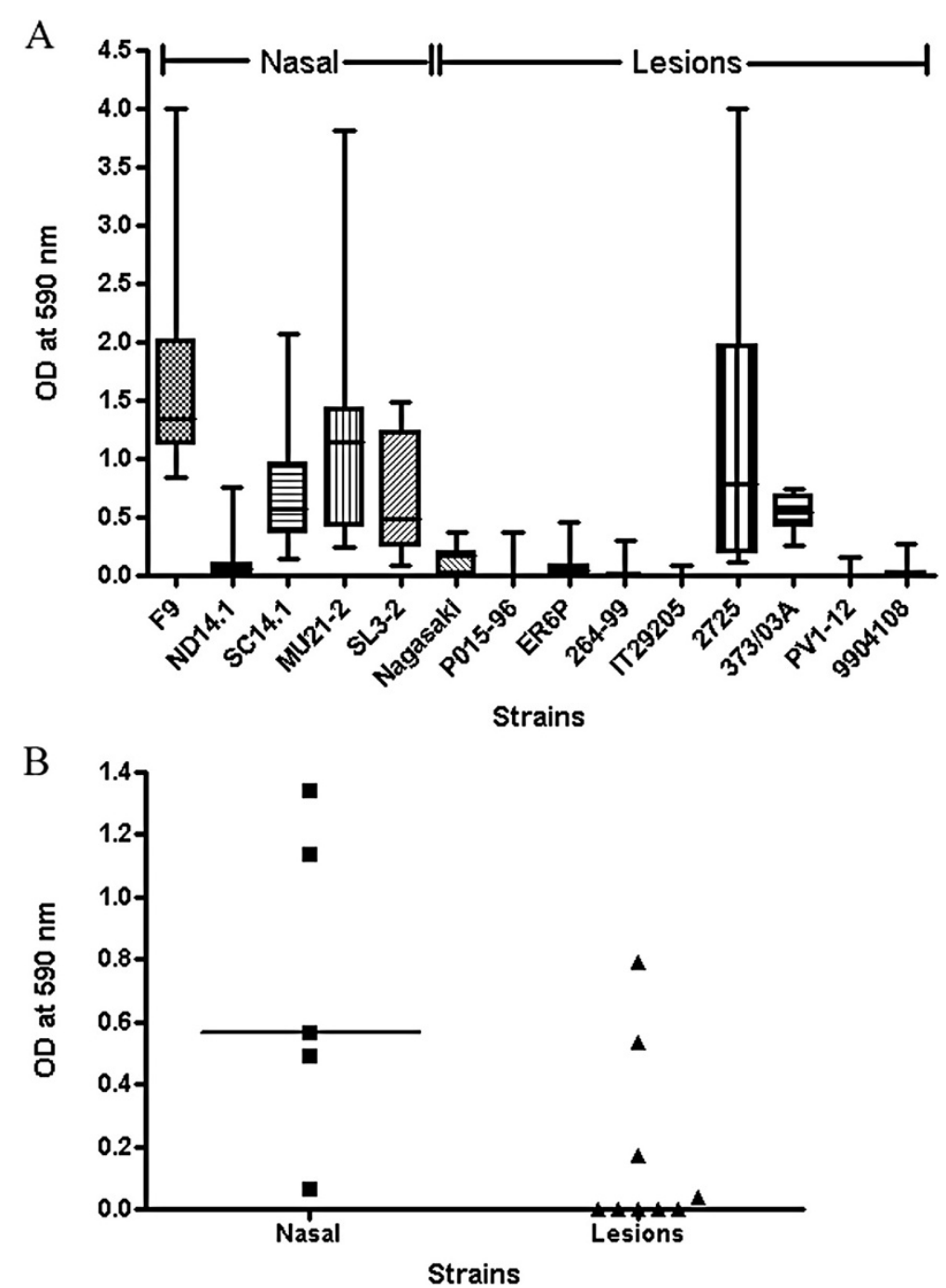

Figure 1 Biofilm formation by H. parasuis isolates. (A) Biofilm formation under static conditions in microtiter plates for Haemophilus parasuis nasal strains $(n=5)$ and strains isolated from lesions of pigs with Glässer's disease $(n=9)$. (B) Medians of biofilm formation for $H$. parasuis strains isolated from the nasal cavities of healthy pigs $(n=5)$ or for strains isolated from the lesions of pigs with Glasser's disease $(n=9)$. Difference between the median of the two groups of strains was statistically significant $(p<0.05)$.

Confocal laser scanning microscopy using different fluorescent probes was performed with the nasal, highbiofilm producer strains F9 and MU21-2 and the weakbiofilm producer virulent strains Nagasaki and ER-6P. The biofilm cells were stained with Film Tracer $^{\mathrm{Tm}} \mathrm{FM}^{\circ} 1$ 43, which becomes fluorescent once it is inserted in the cell membrane. The biofilms were also stained with fluorescent probes to label poly- $\mathrm{N}$-acetylglucosamine (PGA), proteins, or extracellular DNA that could be present in the biofilm matrix. The data indicated that the biofilm matrix of these strains contains PGA, proteins, and eDNA; strain MU21-2 seemed however to produce less PGA than the other three strains (Figure 2). To further characterize the biofilms, 15 images of biofilm layers were recorded and stacked, and 3D-images of the biofilms were generated (Figure 3). Based on these reconstructions, the thickness as well as the biomass of the biofilm produced by each strain was evaluated. The thickness of the weak-biofilm producer strains Nagasaki and ER-6P was approximately $40 \mu \mathrm{m}$ while the thickness of the high-biofilm producer strains MU21-2 and F9 was $50 \mu \mathrm{m}$ and $70 \mu \mathrm{m}$, respectively. This was in agreement with the biomass $\left(\mu \mathrm{m}^{3} / \mu \mathrm{m}^{2}\right)$ which was 17 for the weakbiofilm producer strain Nagasaki while the biomass of the high-biofilm producer strain F9 was 34 .

Biofilms of strains F9, MU21-2, Nagasaki and ER-6P were digested with enzymes to further characterize the composition of the matrix. All strains were resistant to dispersin B (more than 70\% of the biofilm remained after treatment) (Figure 4A). All strains were however sensitive 


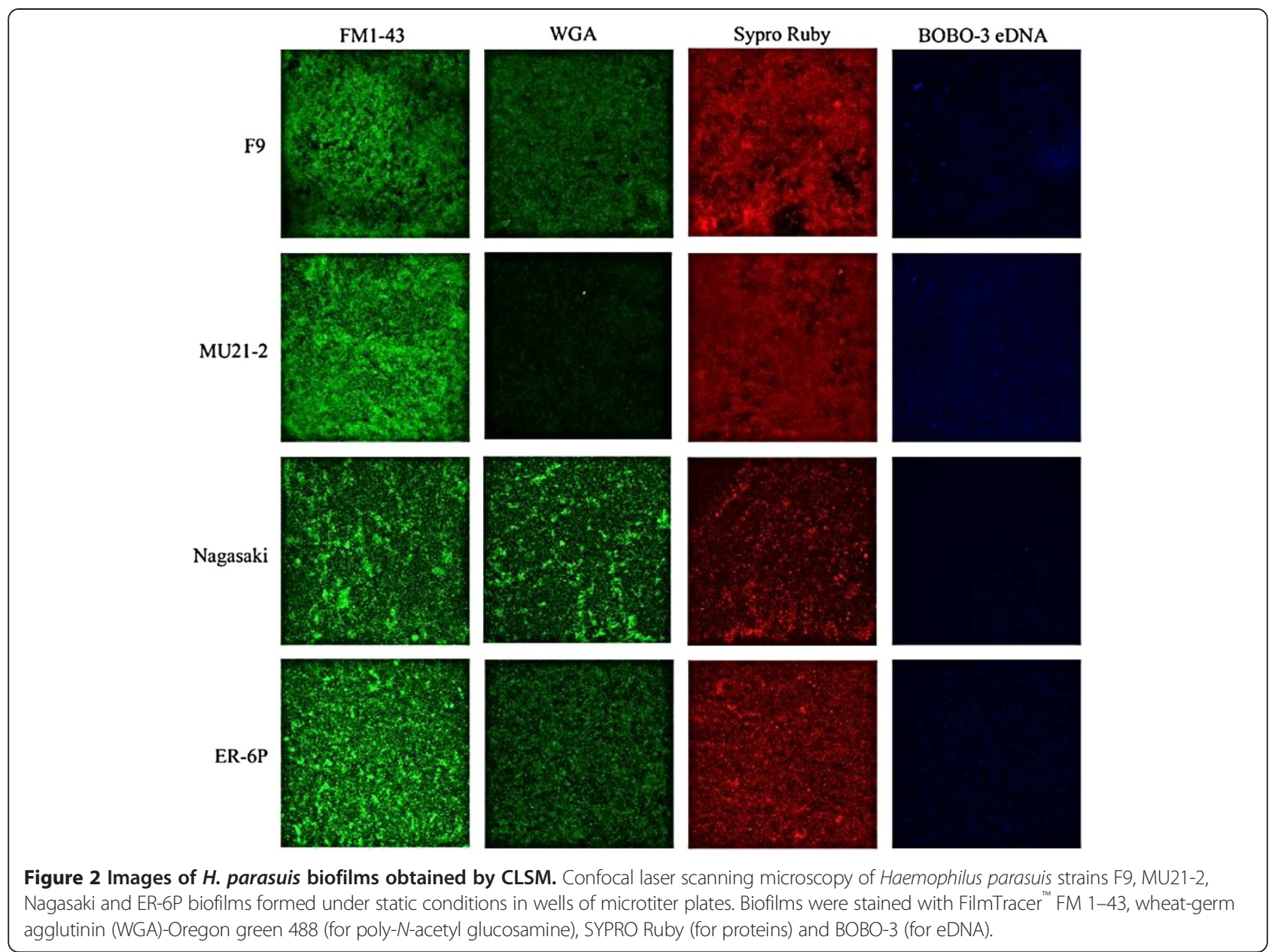

to the proteinase $\mathrm{K}$ treatment (Figure $4 \mathrm{~B}$ ), with strain Nagasaki showing the lowest sensitivity ( $80 \%$ of the biofilm remaining after treatment) and strain ER-6P showing the highest sensitivity (less than $20 \%$ of the biofilm remaining after treatment). Most strains were not affected by DNase I treatment (more than $70 \%$ of the biofilm remained after treatment) except strain F9 which was highly sensitive (with less than $20 \%$ of the biofilm remaining after treatment) (Figure $4 \mathrm{C}$ ). This suggested that proteins, and, in one strain, extracellular DNA

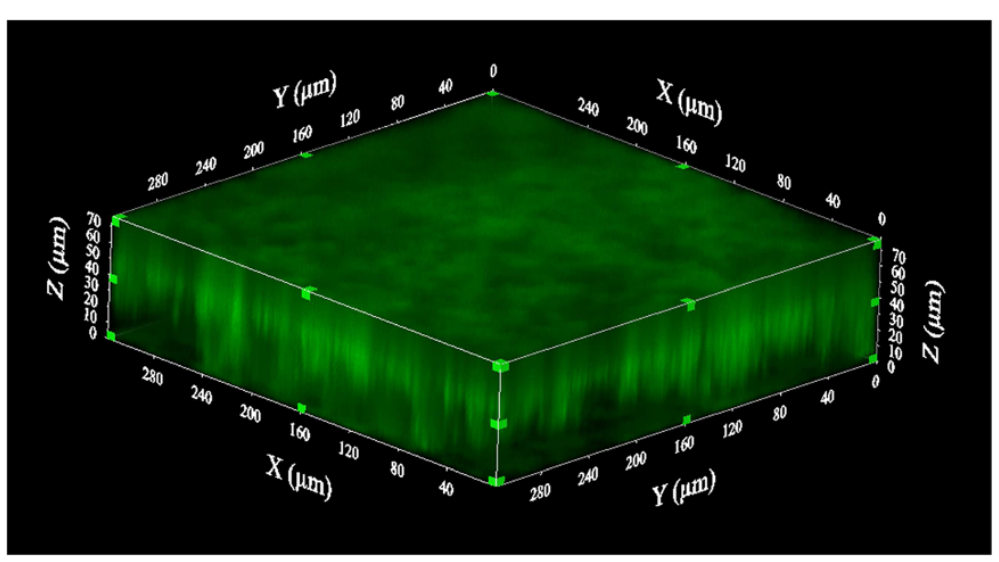

Figure 3 3D images of $\boldsymbol{H}$. parasuis strain F9 obtained by CLSM. Confocal laser scanning microscopy three-dimensional images of Haemophilus parasuis strain F9 biofilm formed under static conditions in wells of a microtiter plate. Biofilm was stained with wheat-germ agglutinin (WGA)-Oregon green 488 . Stack of sections of the $X-Z$ plane of the biofilm. 


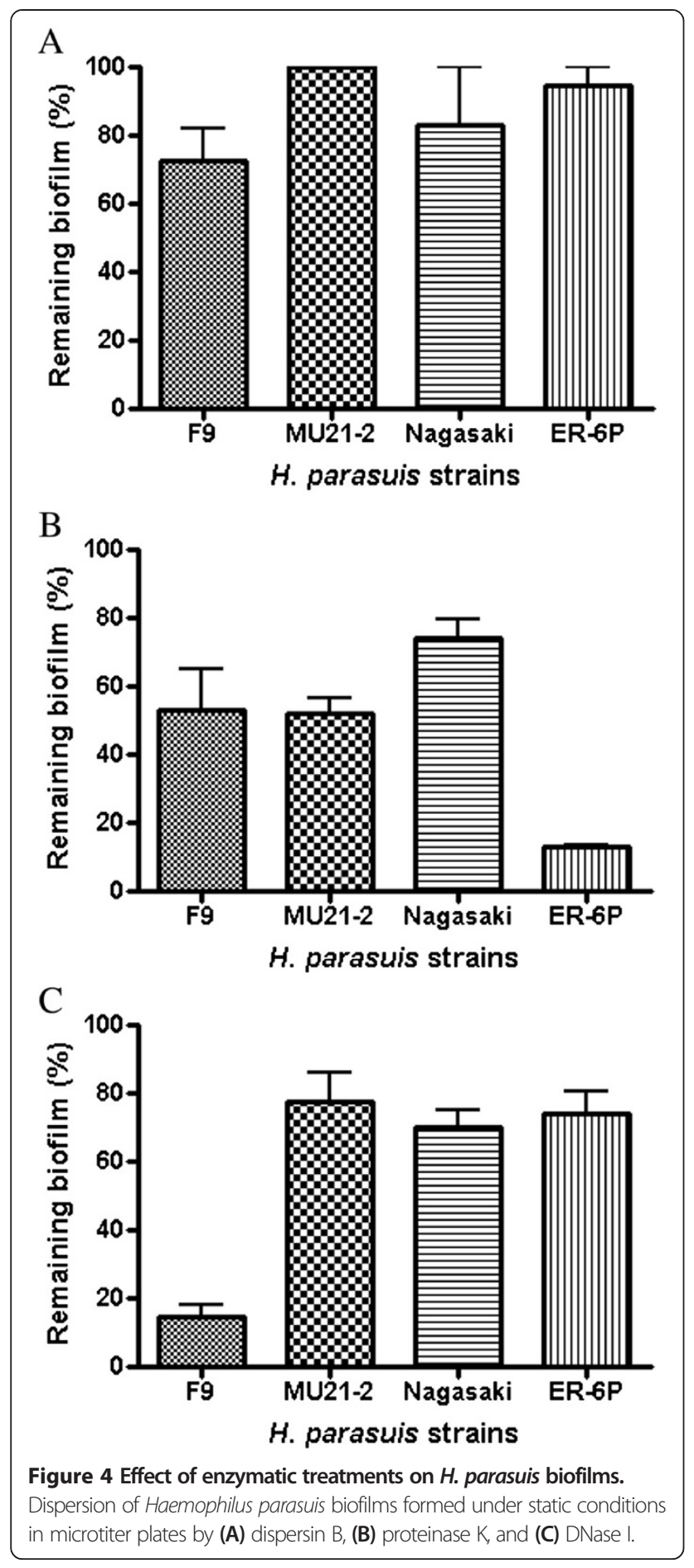

play a larger role than PGA in $H$. parasuis biofilm formation.

Biofilm formation under shear force conditions

Previous experiments with A. pleuropneumoniae and the drip-flow apparatus indicated that this biofilm reactor could be used to grow biofilm of fastidious Pasteurellaceae isolated from the upper respiratory tract [24]. The conditions (50 mL/channel/hour; 50\% BHI-NAD) used to grow A. pleuropneumoniae biofilms in the drip-flow apparatus were used for the first test. However, these did not support the growth of $H$. parasuis biofilms. Given that $H$. parasuis is more fastidious than A. pleuropneumoniae, a full strength BHI-NAD was used and the speed of the flow was reduced by half. These conditions allowed the two strains with a strong-biofilm phenotype, MU21-2 and F9, to produce visible biofilms (Figure 5). Although a thin film was observable for Nagasaki and ER-6P, which are strains with a weak-biofilm phenotype under static conditions, the conditions of the drip-flow apparatus did not enhance the biofilm formation by these strains (Figure 5). As indicated by the biofilm dry weight, strain MU21-2 produced a larger biomass than strain F9 under these flow conditions (Table 2).

The BioFlux 200 flowthrough device is a high throughput microfluidic system that has been recently tested for the growth of bacterial biofilms [25]. This system has yet to be tested with Pasteurellaceae and could provide certain advantages over the drip-flow apparatus. For example, the system requires smaller volumes, which range in $\mu \mathrm{L}$ to $\mathrm{mL}$, and can be used for high throughput screens. Initial density of the inoculum $\left(\mathrm{OD}_{600}\right.$ of 1.0, 0.5 and 0.25$)$ and the incubation time ( $2 \mathrm{~h}$ and $4 \mathrm{~h}$ ) for the initial attachment were the first parameters tested with a relatively low shear force $\left(0.5\right.$ dyne $\left./ \mathrm{cm}^{2}\right)$. An $\mathrm{OD}_{600}$ of 0.25 and an incubation time of $2 \mathrm{~h}$ for the initial attachment were sufficient for strains MU21-2 and F9 to form biofilms that block the microfluidic channel after 6 h (Figure 6); however, strains Nagasaki and ER-6P did not form biofilms (Figure 6). To prevent blocking of the channel, a range of shear force was tested with an inoculum at $\mathrm{OD}_{600}$ of 0.25 and an incubation time of $2 \mathrm{~h}$ for the initial attachment. Both strains MU21-2 and F9 were not able to form a biofilm when the shear force was equal or above $0.7 \mathrm{dyne} / \mathrm{cm}^{2}$. Between 0.5 and $0.7 \mathrm{dyne} / \mathrm{cm}^{2}$, both strains always blocked the channel within $12 \mathrm{~h}$ but it took longer as the shear force was increased. Therefore, in our hands, this microfluidic system can only be used to study biofilm formation of $H$. parasuis during short incubation periods.

\section{Effect of fibrinogen and fibronectin on biofilm formation} It has been shown that supplementing the culture medium with fibrinogen induces biofilm formation of Streptococcus suis, another important swine pathogen [46]. Thus, we evaluated the effect of supplemental fibrinogen on $H$. parasuis biofilm formation. As shown in Figure 7, fibrinogen (at a concentration of $1 \mathrm{mg} / \mathrm{mL}$ ) inhibited biofilm formation by all four strains. Fibronectin had no effect on bioflm formation (data not shown). Inhibition of biofilm formation by fibrinogen was not related to an inhibition of growth since fibrinogen did not affect growth of $H$. parasuis (data not shown). 


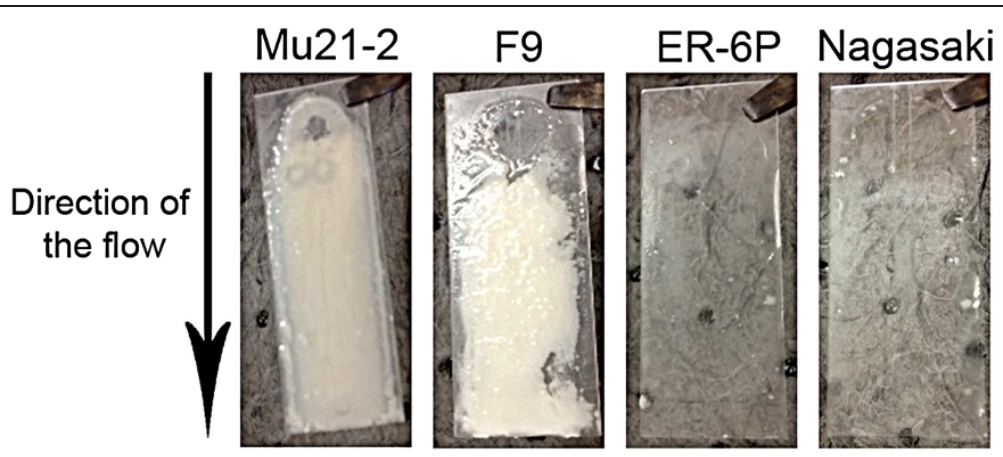

Figure 5 Biofilm formation by $\boldsymbol{H}$. parasuis in a drip-flow apparatus. Biofilm formation under low shear force in a drip-flow apparatus. Images of typical biofilms for Haemophilus parasuis strains MU21-2, F9, ER-6P, and Nagasaki visible after $24 \mathrm{~h}$ of incubation with continuous flow (25 mL/h).

Transcriptional profile of $H$. parasuis F9 grown in biofilm Given that the genome of strain F9 was not previously sequenced, the genome of this high-biofilm producer was sequenced to facilitate transcriptional analysis. The assembly size of the F9 genome was $2.49 \mathrm{Mb}$, with an n50 of 44 023 and 644 contigs, with a $\mathrm{G}+\mathrm{C}$ content of $39 \%$, which is comparable to that of the draft and complete genomes of $H$. parasuis [30,47]. Transcriptomic analysis was performed with an average of $2 \mathrm{M}$ sequence reads per mRNA sample. More than $75 \%$ of the reads for each sample were mapped. The majority of reads mapped with a mapping quality $(\mathrm{MAPQ}) \geq 20$, and only reads mapping with a $M A P Q \geq 10$ were further processed for differential gene expression analysis (Additional file 2A). Some reads mapped in non-protein coding sequences, mainly in tRNA gene sequences, and were particularly high in the stationary culture sample (Additional file 2B). Differential expression analysis found 425 DEGs in biofilm (B) when compared to planktonic growth (P) (Table 3). When B or $\mathrm{P}$ condition was compared to the stationary-phase culture (S), a notable increase in the number of up-regulated genes was observed (Figure 8 and Table 3). Filtered lists of DEGs for B vs P, P vs S and B vs S are shown in Additional files 3,4 and 5 , respectively. A large number of up- and down-regulated genes were shared between B vs $S$ and $\mathrm{P}$ vs $\mathrm{S}$ comparisons (Figure $9 \mathrm{~A}$ and $\mathrm{B}$ ), although a considerable amount was unique to each condition. Fifty-five genes were up-regulated in all three comparisons,

Table 2 Average dry weight (in $\mathrm{mg}$ ) for drip-flow biofilms formed after $24 \mathrm{~h}$ of continuous flow by 4 different strains of Haemophilus parasuis

\begin{tabular}{ll}
\hline Strain & Biofilm dry weight $( \pm$ Standard error of the mean $)$ \\
\hline MU21-2 & $8.33( \pm 3.23)$ \\
F9 & $3.37( \pm 0.38)$ \\
Nagasaki & $\mathrm{ND}^{1}$ \\
ER-6P & $\mathrm{ND}$
\end{tabular}

${ }^{1}$ The amount of biofilm was below the detection limit. which included 8 ribosomal proteins. On the other hand, 56 up-regulated genes were unique to the biofilm and included, among others, six transcriptional regulators, possibly involved in biofilm formation (Additional file 6).

Blast2GO allowed 76\% of GO term assignment to $H$. parasuis proteome, with a good GO level distribution (mean level $=6.8 ; \mathrm{SD}=2.7$ ) and more than $8 \mathrm{~K}$ annotations. Enrichment analysis of the DEGs $(P<0.05)$ identified a large number of up- or down-regulated pathways

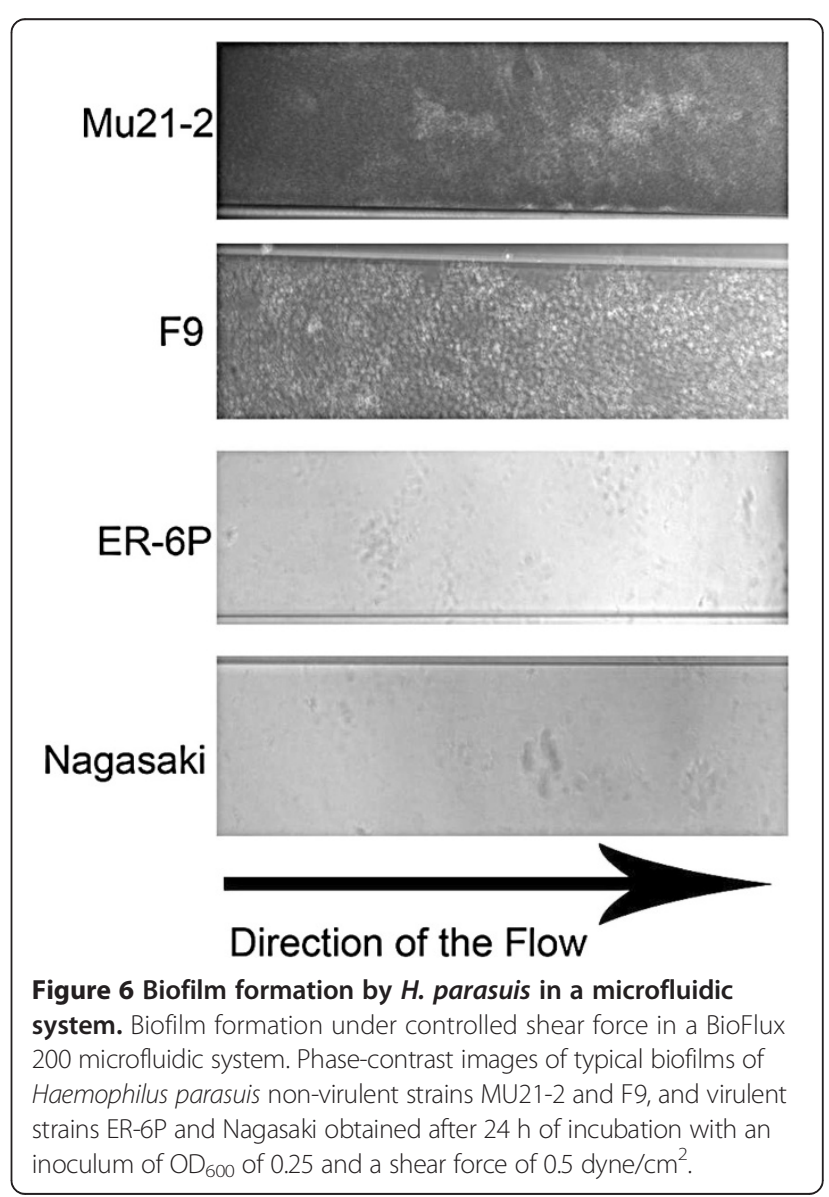




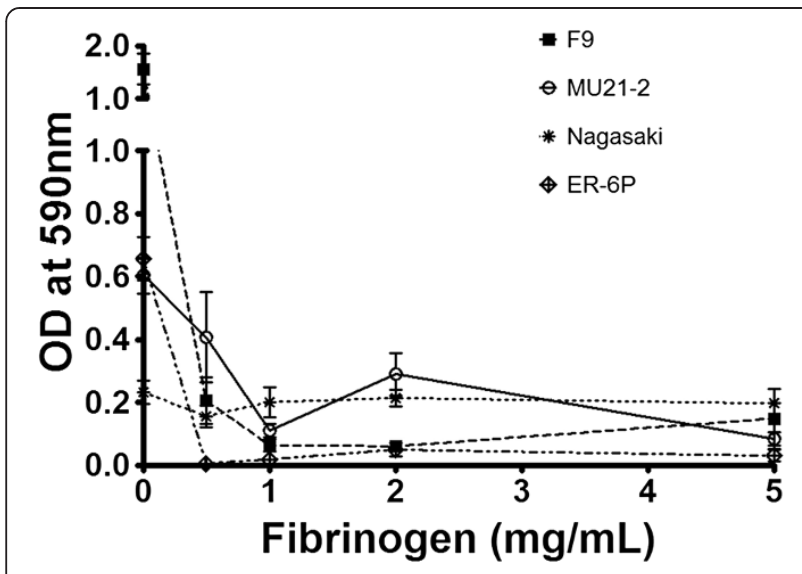

Figure 7 Effects of fibrinogen on biofilm formation by $\mathrm{H}$. parasuis. Effects of various concentrations of fibrinogen added to the culture medium on biofilm formation by Haemophilus parasuis strains F9, MU21-2, Nagasaki and ER-6P under static conditions in microtiter plates. Assays were performed in triplicate, and the means \pm standard deviations are indicated.

(Additional file 7). Analysis was visualized performing Venn diagrams (Additional files 8 and 9). All three comparisons shared the enriched up-regulated GO term "structural constituent of ribosome". Enrichment of B vs S and $\mathrm{P}$ vs $\mathrm{S}$ shared the following GO terms: activation of response to oxidative stress, iron ion binding, ribosome biogenesis, unfolded protein binding and peroxidase activity pathways, indicating the importance of these functions for both biofilm and planktonic growth. Interestingly, protein folding, cell outer membrane, protein secretion, and sequence-specific DNA binding (transcriptional regulators) GO terms were up-regulated specifically in B vs $S$ and $\mathrm{B}$ vs $\mathrm{P}$ comparisons, which could indicate specific biofilm pathways. Other GO terms were specific to each comparison. A total of 20 up-regulated GO terms were specific to $\mathrm{B}$ vs $\mathrm{P}$ comparison, such as lipopolysaccharide transport, response to stress or DNA-mediated regulation of transcription (transcriptional regulators) (Additional file 9). The same analysis was performed for downregulated genes and showed that membrane-related genes were over-represented among these genes in all

Table 3 Summary of the differential expression analysis performed with edgeR tool $(P$-value $<0.05)$

\begin{tabular}{lllll}
\hline Comparison & Up & Down & Total & ${ }^{4}$ Genome (\%) \\
\hline${ }^{1}$ B vs ${ }^{2} P$ & 212 & 213 & 425 & 19 \\
$B$ B v ${ }^{3}$ S & 538 & 571 & 1109 & 49 \\
P vs S & 376 & 417 & 793 & 35 \\
\hline
\end{tabular}

${ }^{1}$ Biofilm.

${ }^{2}$ Planktonic.

${ }^{3}$ Stationary phase.

${ }^{4} 2259$ of the 2317 annotated protein-coding genes were taken as total

(at least one count per million (cpm) in at least two samples).

Percentages of differentially expressed genes are also shown for each comparison. three comparisons. These membrane-related genes were related to transport, more especially to sodium ion transport or phosphoenolpyruvate-dependent sugar phosphotransferase system (PTS). B vs $\mathrm{S}$ and $\mathrm{P}$ vs $\mathrm{S}$ detected down-regulated genes related to nutrient transport, such as ions, amino acids or monosaccharides, which indicate common pathways in biofilm and planktonic conditions. On the other hand, some enriched GO terms were specific to biofilm, as evidenced by the B vs P comparisons. For instance, GOs related to translation (ribosomal proteins) were among the most significantly down-regulated, which suggest a possible low metabolic state, but these enriched GO terms were caused by different ribosomal proteins than the ones responsible for the shared enriched upregulated GO term (Additional file 9).

The F9 surface-associated proteins were predicted using Phobius and the conservation of the up-regulated ones was examined by tBLASTn in the $14 \mathrm{H}$. parasuis genomic sequences available in GenBank. The number of predicted membrane proteins up-regulated in $\mathrm{F} 9$ grown under biofilm conditions (B vs S) was 148 from a total of 538. The majority of the predicted membrane proteins were well conserved among the majority of strains, including a $28 \mathrm{kDa}$ outer membrane protein (HS372_00711), Omp P5 precursor (HS372_01222), PilQ (HS372_02002) and Omp85 (HS372_00165) (Additional file 10). Others were found exclusively in non-virulent isolates, such as OmpW (HS372_00498), prophage CP457 integrase (HS372_00596), filamentous hemagglutinin FhaB (HS372_01074) or five hypothetical proteins (HS37 2_00147, HS372_01332, HS372_02390, HS372_00611 and HS372_02391) (Additional file 10). Additionally, FhaC (annotated as TpsB) was also found only in the nonvirulent strains (Additional file 10).

$H$. parasuis gene expression during biofilm and planktonic growth was compared to transcriptomic data of $A$. pleuropneumoniae, another swine pathogen member of the Pasteurellaceae family, from a previous study [24]. Although preliminary analysis showed that A. pleuropneumoniae and $H$. parasuis F9 shared only $60 \%$ of the genome, some common up-regulated genes were found under biofilm condition (Table 4), but no predominant pathways were detected, suggesting different regulatory networks for these two species. Notably, proteins related to anaerobic metabolism such as cytochrome c-type protein NapC (HS372_02085) or putative electron transport protein yccM (HS372_02091) and some lipoproteins (HS372_01222 and HS372_00366) were found in both bacteria.

\section{Discussion}

In the present study, we used well-characterized virulent and non-virulent strains of $H$. parasuis from our collection [13]. We found that most nasal strains (4/5) were 

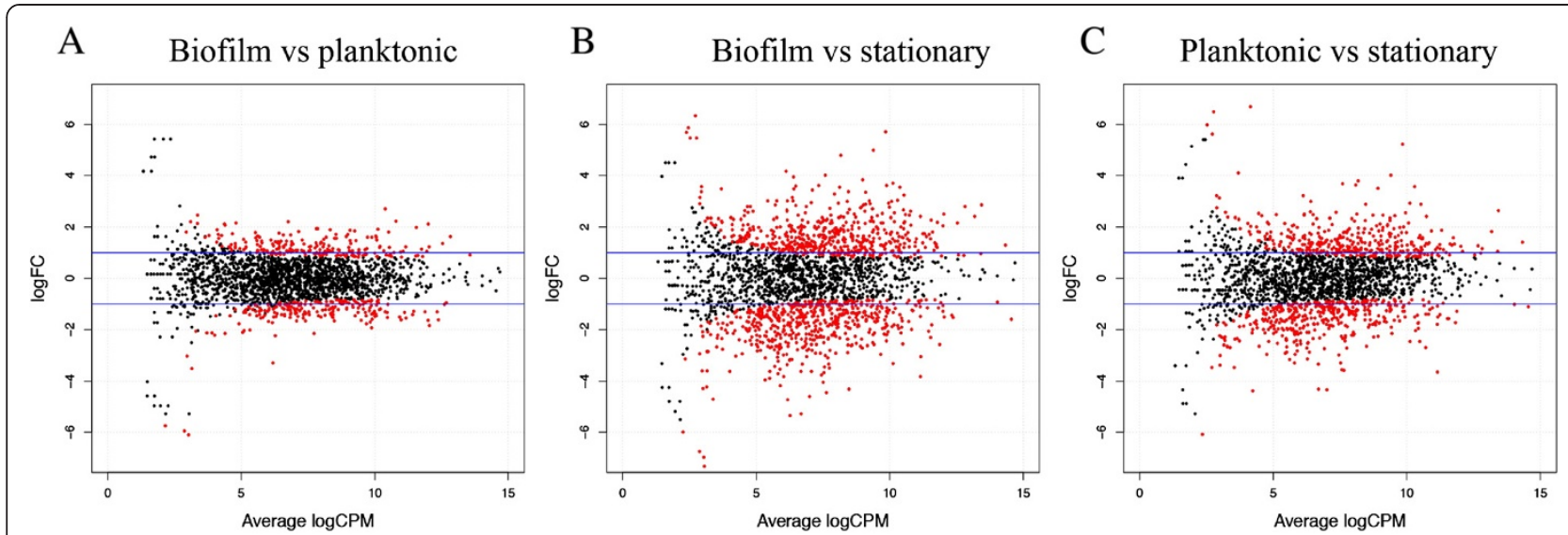

Figure 8 Identification of $\boldsymbol{H}$. parasuis genes differentially expressed. MA plots generated by EdgeR showing transcript expression profiles in the three comparisons performed: biofilm vs planktonic (A), biofilm vs stationary phase (B) and planktonic vs stationary phase (C). For each gene, $\log _{2}$ (fold change) between the two conditions is plotted ( $M, y$ axis) against the gene's $\log _{2}$ (average expression) in the two samples ( $A, x$ axis). The blue lines indicate 2 -fold changes. Red dots highlight the genes at $5 \%$-value.

strong-biofilm producers whereas most systemic strains (8/9) formed no biofilm or were very weak-biofilm producers. Similarly, a strong association was found between MLST clusters [2] and biofilm formation. Strains of MLST cluster $\mathrm{C}$ (which are associated with nasal isolation) formed more biofilms than strains of MLST cluster A (which are isolated from systemic lesions). Similarly, the presence of autotransporter $v t a A$ group 1 genes and of sialyltransferase $l s g B$ gene associated with virulent strains $[13,48]$, and was not associated with strong biofilm formation. Overall, our results are in agreement with Jin et al. [19] who observed that, generally, serovars considered non-virulent showed a higher degree of biofilm formation than virulent serovars. Although it is important to note that a strict relationship between serovars and virulence in H. parasuis has not been demonstrated [1].
The use of CLSM and fluorescent probes showed the presence of PGA, proteins, and eDNA in the biofilm matrix of $H$. parasuis nasal, high-biofilm producer strains F9 and MU21-2 and of the weak-biofilm producer strains Nagasaki and ER-6P recovered from lesions of pigs with Glässer's disease. This is, to the best of our knowledge, the first report of the presence of PGA and proteins in the biofilm matrix of $H$. parasuis. PGA is the major component of the biofilm matrix of several bacteria including other Pasteurellaceae members such as A. pleuropneumoniae and Aggregatibacter actinomycetemcomitans $[18,49]$. Nevertheless all 4 strains tested were resistant to dispersin B. All strains were however sensitive to a proteinase $\mathrm{K}$ treatment suggesting that proteins play a larger role than PGA in H. parasuis biofilm formation. Tang et al. [50] have shown that treatment with the staphylococcal
A UP

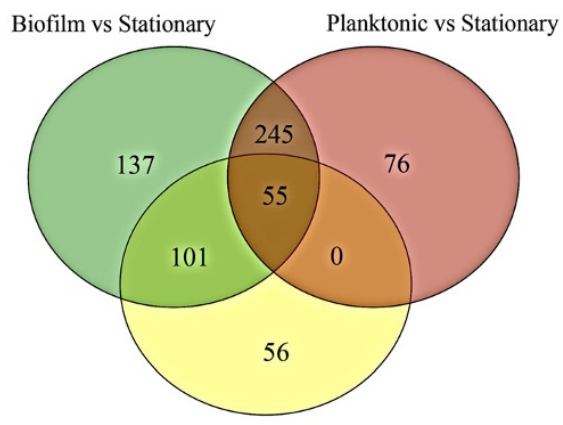

Biofilm vs Planktonic
B

DOWN

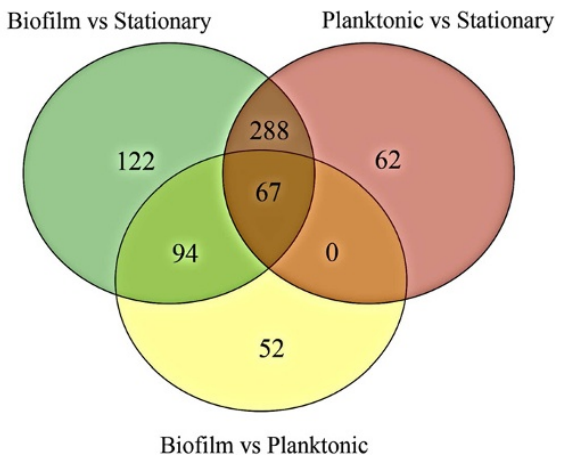

Figure 9 Comparison of $\boldsymbol{H}$. parasuis genes that were up- or down-regulated. Venn diagrams of Haemophilus parasuis genes identified as up- (A) and down-regulated (B) under different growth states. 
Table 4 Common up-regulated genes between Haemophilus parasuis $\mathrm{F} 9$ and Actinobacillus pleuropneumoniae when grown in biofilm condition

H. parasuis (this study) vs A. pleuropneumoniae [22]

\begin{tabular}{lll}
\hline $\begin{array}{l}\text { H. parasuis } \\
\text { F9 }\end{array}$ & App & Product \\
\hline HS372_02083 & APL_1425 & Cytochrome c-type protein NapC \\
HS372_02085 & APL_1427 & Putative electron transport protein yccM \\
HS372_02091 & APL_1821 & 50S ribosomal protein L31 type B \\
HS372_00945 & APL_1440 & $\begin{array}{l}\text { High-affinity zinc uptake system protein znuA } \\
\text { precursor }\end{array}$ \\
HS372_00147 & APL_1894 & hypothetical protein \\
HS372_02009 & APL_1423 & Putative esterase \\
HS372_02012 & APL_0433 & Peptide methionine sulfoxide reductase MsrB
\end{tabular}

HS372_02012 APL_0433 Peptide methionine sulfoxide reductase MsrB

HS372_01222 APL_0460 Outer membrane protein P5 precursor

HS372_00060 APL_1206 putative ribonuclease FitB

HS372_01900 APL_1173 Nicotinamide riboside transporter pnuC

HS372_00666 APL_0442 vancomycin high temperature exclusion protein

HS372_01587 APL_0484 Alpha-aminoadipate-lysW ligase lysX

HS372_02062 APL_0038 hypothetical protein

HS372_00364 APL_0222 Putative lipoprotein/NMB1162 precursor

HS372_01892 APL_0133 Cys regulon transcriptional activator

HS372_00988 APL_1295 Arginine repressor

HS372_02252 APL_1873 Succinyl-diaminopimelate desuccinylase

HS372_02064 APL_0036 hypothetical protein

HS372_01893 APL_0134 hypothetical protein

HS372_01521 APL_1320 Thiamine import ATP-binding protein ThiQ

HS372_02387 APL_1059 Integrase core domain protein

HS372_00061 APL_1207 prevent-host-death family protein

HS372_00916 APL_0423 Ribonuclease HI

HS372_01200 APL_0593 Inosine-5'-monophosphate dehydrogenase

HS372_01950 APL_1574 Putative hydrolase ydeN

HS372_02244 APL_0254 Cytosol non-specific dipeptidase

HS372_01281 APL_1230 Phosphoserine phosphatase

HS372_01385 APL_0967 Glutamate permease

HS372_01220 APL_0463 Putative phosphinothricin acetyltransferase YwnH

HS372_01490 APL_0928 hypothetical protein

HS372_01179 APL_1499 Threonine synthase

HS372_02366 APL_0395 Sigma-E factor negative regulatory protein

HS372_01208 APL_0895 Formate dehydrogenase-N subunit gamma

HS372_01221 APL_0461 putative phosphatase YwpJ

HS372_02265 APL_0687 D-lactate dehydrogenase

HS372_01342 APL_1448 Spermidine/putrescine import ATP-binding protein PotA

HS372_00366 APL_0220 Putative lipoprotein/NMB1164 precursor

HS372_00099 APL_1853 Ketol-acid reductoisomerase nuclease NUC1 decreased slightly the biofilm formation of $H$. parasuis strain 0322. This is in agreement with results of this study showing the presence of eDNA in the biofilm matrix of $H$. parasuis and the high sensitivity of strain F9 biofilm to DNase I digestion.

This is also the first description of $H$. parasuis biofilm formation under shear force in a drip-flow reactor and a microfluidic system. Interestingly, similar biofilm phenotypes (i.e. high-producer vs weak-producer) were observed when static or controlled flow conditions were used. The drip-flow apparatus is a system that it is thought to create an environment with an air-liquid interface which closely resembles the lung environment [23]. Under the conditions we selected, the two strains with a strong-biofilm phenotype, MU21-2 and F9, produced visible biofilms while a thin film was observable for Nagasaki and ER-6P, which are strains with a weak-biofilm phenotype under static conditions. We also used the BioFlux flowthrough device, a high throughput microfluidic system that has been recently tested for the growth of dental plaque bacteria biofilms [51] and Pseudomonas aeruginosas biofilms [25]. Again, strains Nagasaki and ER-6P did not form biofilms under the conditions tested but strains MU21-2 and F9 formed biofilms that rapidly blocked the microfluidic channel. This microfluidic system can therefore be used to study biofilm formation of $H$. parasuis but for short incubation periods. This potentially can be used to investigate genes involved in the early steps of biofilm formation and, if the appropriate tools are combined, to study real-time gene expression during the early steps as demonstrated in Staphylococcus aureus [52].

The genes involved in $H$. parasuis biofilm formation are currently not known. Recently, it was reported that both galU and galE genes seemed to play a role in biofilm formation of $H$. parasuis [12]. A galU mutant was unable to form biofilm in a glass tube while a galE mutant produced more biofilm than the parent wild-type strain. Surprisingly however, the galu mutant also showed an increased tendency to autoagglutinate which is usually associated with a greater ability to form biofilm. Here, the transcriptome of $H$. parasuis $\mathrm{F} 9$ strain showed that static biofilm and planktonic cultures are in similar biological states but not identical, whereas greater differences in gene expression were evident when compared to stationary-phase culture. Subtle differences in gene expression between biofilm and planktonic cells have been also reported for other bacterial species [53,54]. In addition to identifying DEGs related to metabolism, gene enrichment allowed the identification of a large proportion of membrane-related genes among the up-regulated genes in biofilm, including some that have been reported for other bacterial species [53,54]. The conservation of some highly expressed membrane-protein genes in biofilms among the $14 \mathrm{H}$. parasuis genomes available indicates that those may not specifically associate 
with biofilm formation. A sub-set of these up-regulated membrane-protein genes, such as the type IV pilus biogenesis protein pilQ, may be involved in adhesion to different abiotic and biotic surfaces. Interestingly, some up-regulated genes, such as $f h a B$ and $f h a C$ (tpsB) or ompW, were only found in the genome of non-virulent strains. This finding, together with the fact that non-virulent strains formed stronger biofilms, suggests a possible role of these genes in biofilm formation. In fact, Fha and OmpW, together with type IV pilus, have been shown to play a role in biofilm formation in other bacteria [55,56]. Additionally, some lipoproteins, such as lipoprotein Plp4, as well as signs of anaerobic metabolism were evidenced in the biofilms of $H$. parasuis and A. pleuropneumoniae, which may indicate a potential role in biofilm formation of both bacterial species. The function of specific genes in biofilm formation will need further confirmation.

Interestingly, experimental infections in snatch-farrowed, colostrum-deprived piglets showed that the $H$. parasuis strains that proceeded to invade the host were not maintained well in the nasal cavities of the piglets [57]. This suggests changes in the bacteria from a "colonizing state" to an "invasive state", which could be modulated by the ability of each animal to control the infection. In the light of the results of the present study, these changes from a "colonizing state" to an "invasive state" could also be due to the ability of a given bacterial strain to form or not a robust biofilm. We propose that biofilm formation might allow the non-virulent strains to colonize and persist in the upper respiratory tract of pigs. Conversely, the predominant planktonic state of the virulent strains might allow them to disseminate within the host. This latter statement is supported by the inhibition of biofilm formation by fibrinogen. It is worth noting that a recent study on the human pathogen Streptococcus pneumoniae showed that biofilm formation in vivo is associated with reduced invasiveness and a dampened cytokine response [58]. High-biofilm production phenotype might therefore not always be linked to virulence.

\section{Additional files}

Additional file 1: Haemophilus parasuis biofilm formation under static conditions in microtiter plates. (A) Medians of biofilm formation for strains that are sensitive or show intermediate resistance to serum $(n=9)$ or for strains that are resistant to serum $(n=5)$. (B) Medians of biofilm formation for strains negative $(n=5)$ or positive $(n=9)$ for vtaA group 1 genes. (C) Medians of biofilm formation for strains belonging to MLST cluster C $(n=5)$ or strains belonging to MLST cluster A $(n=7)$. (D) Medians of biofilm formation for strains negative $(n=8)$ or positive $(n=6)$ for the sialyltransferase gene $1 \mathrm{sg} B$. Differences between the median of the two groups of strains were not statistically significant $(\mathbf{A}: p=0.059$; B: $p=0.189 ; \mathbf{C}: p=0.202 ; \mathbf{D}: p=0.228)$.

Additional file 2: Mapping of RNA sequencing reads to the H. parasuis strain F9 genome. Overview of the mapping (A) and read counts (B) results. Mapping quality (MAPQ) shows that most of the reads were aligned with $M A P Q \geq 20$ but a considerable percentage of reads were not taken into account for differential expression because mapping to non-protein coding regions, particularly for stationary culture sample.

Additional file 3: List of $H$. parasuis strain F9 genes differentially expressed in biofilm vs planktonic cells. Differentially expressed genes in biofilm versus planktonic $H$. parasuis cells (FDR $<0.05$ ). Sorted by logFC. Actinobacillus pleuropneumoniae (App) first BLASTp hits are also shown.

Additional file 4: List of $H$. parasuis strain F9 genes differentially expressed in planktonic vs stationary phase cells. Differentially expressed genes in planktonic versus stationary $H$. parasuis cells (FDR $<0.05$ ). Sorted by logFC. Actinobacillus pleuropneumoniae (App) first BLASTp hits are also shown.

Additional file 5: List of $H$. parasuis strain F9 genes differentially expressed in biofilm vs stationary phase cells. Differentially expressed genes in biofilm versus stationary $H$. parasuis cells (FDR $<0.05$ ). Sorted by logFC. Actinobacillus pleuropneumoniae (App) first BLASTp hits are also shown.

Additional file 6: List of $\boldsymbol{H}$. parasuis strain F9 up-regulated genes or unique to biofilm. Haemophilus parasuis F9 genes up-regulated in all three comparisons (biofilm vs planktonic; biofilm vs stationary phase; planktonic vs stationary phase) or unique to biofilm.

Additional file 7: List of gene ontology (GO) terms following enrichment analysis of $H$. parasuis strain F9 differentially expressed genes. Enriched Gene Ontology biological process and cellular component nodes among up-regulated genes (FDR $<0.001$ ). No enriched CC GO terms were found for biofilm vs planktonic comparison.

Additional file 8: Visualization of gene ontology (GO) terms following enrichment analysis of $H$. parasuis strain F9 differentially expressed genes. Venn diagrams of Haemophilus parasuis enriched Gene Ontology (GO) terms among the subsets of differentially expressed genes identified as up- (A) and down-regulated (B) under different growth states. Only most specific GO terms are shown.

Additional file 9: List of $H$. parasuis strain F9 GO terms shown in Additional file 8 diagrams. H. parasuis F9 GO terms results from Additional file 8 Venn diagrams.

Additional file 10: List of $H$. parasuis strain F9 membrane-related genes differentially expressed in biofilm compared to planktonic or stationary phase cells. Conservation of Haemophilus parasuis F9 membrane-related genes differentially expressed $(P<0.05)$ in biofilms vs stationary culture or biofilms vs planktonic culture among $14 \mathrm{H}$. parasuis isolates. Genes are sorted by logFC. *Truncated.

\section{Competing interests}

The authors declare that they have no competing interests.

\section{Authors' contributions}

$\mathrm{BBO}$ carried the transcriptomics experiments, and participated in the writing of the manuscript. VD carried the static biofilm formation assays, and participated in the writing of the manuscript. YDNT carried the biofilm formation assays under shear force, and participated in the writing of the manuscript. JL carried the confocal microscopy, the enzymatic treatments and the statistical analysis, and participated in the writing of the manuscript. $\mathrm{KJH}, \mathrm{AWT}$ and DJM sequenced and assembled the genome of strain F9 and participated in the writing of the manuscript. VA conceived the study, participated in its design, and participated in the writing of the manuscript. $\mathrm{MJ}$ conceived the study, participated in its design, and drafted the manuscript. All authors read and approved the final manuscript.

\section{Acknowledgements}

This work was supported in part by a discovery grant from the Natural Sciences and Engineering Research Council of Canada to MJ and by a grant AGL201015232 from the Ministerio de Economía y Competitividad of Spain to VA. VD was a recipient of a doctoral scholarship and of an international training award from Fonds de recherche du Québec - Nature et technologies. BBO is a recipient of a FPI fellowship from the Spanish Ministerio de Economía y Competitividad. The authors would also like to acknowledge the support of the Gouvernement du Québec, Ministère des Relations internationales, de la Francophonie et du Commerce extérieur $\left(V \|^{\mathrm{e}}\right.$ rencontre du Comité mixte Québec-Catalogne, projet 07.304). 


\section{Author details}

${ }^{1}$ Centre de Recerca en Sanitat Animal (CReSA), UAB-IRTA, Campus de la Universitat Autònoma de Barcelona, 08193 Bellaterra, Cerdanyola del Vallès, Spain. ${ }^{2}$ Groupe de recherche sur les maladies infectieuses du porc, Faculté de médecine vétérinaire, Université de Montréal, St-Hyacinthe, Québec J2S 7C6, Canada. ${ }^{3}$ Department of Veterinary Medicine, University of Cambridge, Cambridge, UK. ${ }^{4}$ Institut de Recerca i Tecnologia Agroalimentàries (IRTA), Barcelona, Spain.

\section{Received: 30 May 2014 Accepted: 26 September 2014}

Published online: 27 November 2014

\section{References}

1. Aragon V, Segalés J, Oliveira S: Glässer's disease. In Diseases of Swine. 10th edition. Edited by Zimmerman JJ, Karriker LA, Ramirez A, Schwartz KJ, Stevenson GW. Chichester, UK: Wiley-Blackwell; 2012:760-769.

2. Olvera $A$, Cerdà-Cuéllar M, Aragon V: Study of the population structure of Haemophilus parasuis by multilocus sequence typing. Microbiology 2006, 152:3683-3690.

3. Cerdà-Cuéllar M, Aragon V: Serum-resistance in Haemophilus parasuis is associated with systemic disease in swine. Vet J 2008, 175:384-389.

4. Olvera A, Ballester M, Nofrarias M, Sibila M, Aragon V: Differences in phagocytosis susceptibility in Haemophilus parasuis strains. Vet Res 2009, 40:24.

5. Aragon V, Bouchet B, Gottschalk M: Invasion of endothelial cells by systemic and nasal strains of Haemophilus parasuis. Vet J 2010, 186:264-267.

6. Vanier G, Szczotka A, Friedl P, Lacouture S, Jacques M, Gottschalk M: Haemophilus parasuis invades porcine brain microvascular endothelial cells. Microbiology 2006, 152:135-142.

7. Zhang B, Tang C, Liao M, Yue H: Update on the pathogenesis of Haemophilus parasuis infection and virulence factors. Vet Microbiol 2014, 168:1-7.

8. Costa-Hurtado M, Aragon V: Advances in the quest for virulence factors of Haemophilus parasuis. Vet J 2013, 198:571-576.

9. Bouchet B, Vanier G, Jacques M, Gottschalk M: Interactions of Haemophilus parasuis and its LOS with porcine brain microvascular endothelial cells. Vet Res 2008, 39:42.

10. Bouchet B, Vanicer G, Jacques M, Auger E, Gottschalk M: Studies on the interactions of Haemophilus parasuis with porcine epithelial tracheal cells: limited role of LOS in apoptosis and pro-inflammatory cytokine release. Microb Pathog 2009, 46:108-113.

11. Xu C, Zhang L, Zhang B, Feng S, Zhou S, Li J, Zou Y, Liao M: Involvement of lipooligosaccharide heptose residues of Haemophilus parasuis SC096 strain in serum resistance, adhesion and invasion. Vet J 2013, 195:200-204

12. Zou Y, Feng S, Xu C, Zhang B, Zhou S, Zhang L, He X, Li J, Yang Z, Liao M: The role of galU and galE of Haemophilus parasuis SC096 in serum resistance and biofilm formation. Vet Microbiol 2013, 162:278-284.

13. Martínez-Moliner V, Soler-Llorens P, Moleres J, Garmendia J, Aragon V: Distribution of genes involved in sialic acid utilization in strains of Haemophilus parasuis. Microbiology 2012, 158:2117-2124.

14. Pina S, Olvera A, Barcelo A, Bensaid A: Trimeric autotransporters of Haemophilus parasuis: generation of an extensive passenger domain repertoire specific for pathogenic strains. J Bacteriol 2009, 191:576-587.

15. Costa-Hurtado M, Ballester M, Galofré-Milà N, Darji A, Aragon V: VtaA8 and VtaA9 from Haemophilus parasuis delay phagocytosis by alveolar macrophages. Vet Res 2012, 43:57.

16. Costerton JW, Stewart PS, Greenberg EP: Bacterial biofilms: a common cause of persistent infections. Science 1999, 284:1318-1322.

17. Hall-Stoodley L, Costerton JW, Stoodley P: Bacterial biofilms: from the natural environment to infectious diseases. Nat Rev Microbiol 2004, 2:95-108

18. Jacques $\mathrm{M}$, Aragon $\mathrm{V}$, Tremblay YDN: Biofilm formation in bacterial pathogens of veterinary importance. Anim Health Res Rev 2010, 11:97-121.

19. Jin H, Zhou R, Kang M, Luo R, Cai X, Chen H: Biofilm formation by field isolates and reference strains of Haemophilus parasuis. Vet Microbiol 2006, 118:117-123.

20. Jin H, Wan Y, Zhou R, Li L, Luo R, Zhang S, Hu J, Langford PR, Chen H: Identification of gene transcribed by Haemophilus parasuis in necrotic porcine lung through the selective capture of transcribed sequences (SCOTS). Environ Microbiol 2008, 10:3326-3336.

21. Wu C, Labrie J, Tremblay YDN, Haine D, Mourez M, Jacques M: Zinc as an agent for the prevention of biofilm formation by pathogenic bacteria. J Appl Microbiol 2013, 115:30-40.
22. Labrie J, Pelletier-Jacques G, Deslandes V, Ramjeet M, Auger E, Nash JH, Jacques M: Effects of growth conditions on biofilm formation by Actinobacillus pleuropneumoniae. Vet Res 2010, 41:03.

23. Goeres DM, Hamilton MA, Beck NA, Buckingham-Meyer K, Hilyard JD, Loetterle LR, Lorenz LA, Walker DK, Stewart PS: A method for growing a biofilm under low shear at the air-liquid interface using the drip flow biofilm reactor. Nat Protoc 2009, 4:783-788.

24. Tremblay YDN, Deslandes V, Jacques M: Actinobacillus pleuropneumoniae genes expression in biofilms cultured under static conditions and in a drip-flow apparatus. BMC Genomics 2013, 14:364.

25. Benoit MR, Conant CG, lonescu-Zanetti C, Schwartz M, Matin A: New device for high-throughput viability screening of flow biofilms. App/ Environ Microbiol 2010, 76:4136-4142.

26. Tremblay YDN, Lamarche $D$, Chever $P$, Haine $D$, Messier $S$, Jacques $M$ : Characterization of the ability of coagulase-negative staphylococci isolated from the milk of Canadian farms to form biofilms. J Dairy Sci 2013, 96:234-246.

27. Quail MA, Kozarewa I, Smith F, Scally A, Stephens PJ, Durbin R, Swerdlow H, Turner DJ: A large genome centre's improvements to the Illumina sequencing system. Nat Methods 2008, 5:1005-1010.

28. Quail M, Swerdlow H, Turner DJ: Improved protocols for the Illumina genome analyzer sequencing system. Curr Protoc Hum Genet 2009, 18:1-27.

29. Lunter G, Goodson M: Stampy : A statistical algorithm for sensitive and fast mapping of Illumina sequence reads. Genome Res 2011, 21:936-939.

30. Xu Z, Yue M, Zhou R, Jin Q, Fan Y, Bei W, Chen H: Genomic characterization of Haemophilus parasuis $\mathrm{SH} 0165$, a highly virulent strain of serovar 5 prevalent in China. PLoS One 2011, 6:e19631.

31. Martin $\mathrm{M}$ : Cutadapt removes adapter sequences from high-throughput sequencing reads. EMBnet J 2011, 17:10-12

32. Joshi N: UC Davis Bioinformatics Core. https://github.com/ucdavisbioinformatics/sickle.

33. Zerbino DR, Birney E: Velvet: algorithms for de novo short read assembly using de Bruijn graphs. Genome Res 2008, 18:821-829.

34. Gladman S: Velvet Optimiser. http://www.vicbioinformatics.com/ velvetoptimiser.manual.txt.

35. Seemann T: Prokka User Manual. http://bioinformatics.net.au/prokka-manual.html.

36. Oliveira S, Galina L, Pijoan C: Development of a PCR test to diagnose Haemophilus parasuis infections. J Vet Diagn Invest 2001, 13:495-501.

37. FastQC: http://www.bioinformatics.babraham.ac.uk/projects/fastqc/

38. FASTX-Toolkit: [http://hannonlab.cshl.edu/fastx_toolkit/]

39. Torrent Mapping Alignment Program: [https://github.com/iontorrent/TMAP]

40. Li H, Handsaker B, Wysoker A, Fennell T, Ruan J, Homer N, Marth G, Abecasis $\mathrm{G}$, Durbin $\mathrm{R}$ and 1000 Genome Project Data Processing Subgroup: The sequence alignment/map (SAM) format and SAMtools. Bioinformatics 2009, 25:2078-2079.

41. Thorvaldsdóttir H, Robinson JT, Mesirov JP: Integrative Genomics Viewer (IGV): high-performance genomics data visualization and exploration. Brief Bioinform 2013, 14:178-192.

42. HTSeq v0.5.4p3: [https://pypi.python.org/pypi/HTSeq]

43. Robinson MD, McCarthy DJ, Smyth GK: edgeR: a Bioconductor package for differential expression analysis of digital gene expression data. Bioinformatics 2010, 26:139-140.

44. Conesa A, Götz S, Garcia-Gomez JM, Terol J, Talon M, Robles M: Blast2GO: a universal tool for annotation, visualization and analysis in functional genomics research. Bioinformatics 2005, 21:3674-3676.

45. Käll L, Krogh A, Sonnhammer EL: A combined transmembrane topology and signal peptide prediction method. J Mol Biol 2004, 338:1027-1036.

46. Bonifait L, Grignon L, Grenier D: Fibrinogen induces biofilm formation by Streptococcus suis and enhances its antibiotic resistance. App/ Environ Microbiol 2008, 74:4969-4972.

47. Mullins MA, Register KB, Bayles DO, Dyer DW, Kuehn JS, Phillips GJ: Genome sequence of Haemophilus parasuis strain 29755. Stand Genomic Sci 2011, 5:61-68

48. Olvera A, Pina S, Macedo N, Oliveira S, Aragon V, Bensaid A: Identification of potentially virulent strains of Haemophilus parasuis using a multiplex PCR for virulence-associated autotransporters (vtaA). Vet J 2012, 191:213-218.

49. Kaplan JB, Velliyagounder K, Ragunath C, Rohde H, Mack D, Knobloch JK, Ramasubbu N: Genes involved in the synthesis and degradation of matrix polysaccharide in Actinobacillus actinomycetemcomitans and Actinobacillus pleuropneumoniae biofilms. J Bacteriol 2004, 186:8213-8220.

50. Tang JN, Kang MS, Chen HC, Shi XM, Zhou R, Chen J, Du YW: The staphylococcal nuclease prevents biofilm formation in Staphylococcus 
aureus and other biofilm-forming bacteria. Sci China Life Sci 2011, 54:863-869.

51. Nance WC, Dowd SE, Samarian D, Chludzinski J, Delli J, Battista J, Rickard AH: A high-throughput microfluidic dental plaque biofilm system to visualize and quantify the effect of antimicrobials. J Antimicrob Chemother 2013, 68:2550-2560

52. Moormeier DE, Endres JL, Mann EE, Sadykov MR, Horswill AR, Rice KC, Fey $P D$, Bayles KW: Use of microfluidic technology to analyze gene expression during Staphylococcus aureus biofilm formation reveals distinct physiological niches. Appl Environ Microbiol 2013, 79:3413-3424.

53. Yadav MK, Kwon SK, Cho CG, Park S-W, Chae S-W, Song J-J: Gene expression profile of early in vitro biofilms of Streptococcus pneumoniae. Microbiol Immunol 2012, 56:621-629.

54. Dötsch A, Eckweiler D, Schniederjans M, Zimmerman A, Jensen V, Scharfe M, Geffers R, Häussler S: The Pseudomonas aeruginosa transcriptome in planktonic cultures and static biofilms using RNA sequencing. PLoS One 2012, 7:e31092.

55. Ritter A, Com E, Bazire A, Goncalves Mdos S, Delage L, Le Pennec G, Pineau C, Dreanno $C$, Compère $C$, Durour A: Proteomic studies highlight outermembrane proteins related to biofilm development in the marine bacterium Pseudoalteromonas sp. D41. Proteomics 2012, 12:3180-3192.

56. Irie $Y$, Mattoo $S$, Yuk MH: The Bvg virulence control system regulates biofilm formation in Bordetella bronchiseptica. J Bacteriol 2004, 186:5692-5698.

57. Aragon V, Cerdà-Cuéllar M, Fraile L, Monbarg M, Nofrarias M, Olvera A, Sibila $M$, Solanes D, Segalés J: Correlation between clinico-pathological outcome and typing of Haemophilus parasuis field strains. Vet Microbiol 2010, 142:387-393.

58. Blanchette-Cain K, Hinojosa CA, Suresh Babu RA, Lizcano A, Gonzalez-Juarbe N, Munoz-Almagro C, Sanchez CJ, Bergman MA, Orihuela CJ: Streptococcus pneumoniae biofilm formation is strain dependent, multifactorial, and associated with reduced invasiveness and immunoreactivity during colonization. MBio 2013, 4:e00745-13.

doi:10.1186/s13567-014-0104-9

Cite this article as: Bello-Ortí et al:: Biofilm formation by virulent and non-virulent strains of Haemophilus parasuis. Veterinary Research 2014 45:104.

\section{Submit your next manuscript to BioMed Central and take full advantage of:}

- Convenient online submission

- Thorough peer review

- No space constraints or color figure charges

- Immediate publication on acceptance

- Inclusion in PubMed, CAS, Scopus and Google Scholar

- Research which is freely available for redistribution 